\title{
New Links between Pulsation and Stellar History
}

\author{
Nancy Remage Evans
}

\begin{abstract}
New instrumentation is providing new insights into intermediate mass pulsating Cepheids, particularly about their formation and history. Three approaches are discussed, using space (Hubble and Chandra) and ground-based studies (radial velocities). First, we are conducting a survey of Cepheids with the Hubble Space Telescope Wide Field Camera 3 (WFC3) to identify possible resolved companions (for example Eta Aql) and thus provide constraints on star formation. Followup Xray observations (Chandra and XMM-Newton) can confirm whether possible low mass companions are young enough to be physical companions of Cepheids. In a related study of intermediate mass stars, Chandra X-ray observations of late B stars in $\operatorname{Tr} 16$ have been used to determine the fraction which have X-ray active low mass companions. Finally, the Tennessee State Automatic Spectroscopic Telescope AST and the Moscow University group have obtained velocities of a number of Cepheids. As an example, the orbit of V350 Sgr has been redetermined, providing a new level of accuracy to the orbital velocity amplitude, which is needed for mass determination.
\end{abstract}

\section{Introduction}

This contribution focuses on two aspects of binary Cepheids: information they provide about star formation and about stellar evolution (masses).

Nancy Remage Evans

SAO, 60 Garden St, MS 4 Cambridge MA 02138, USA e-mail: nevansecfa.harvard.edu 

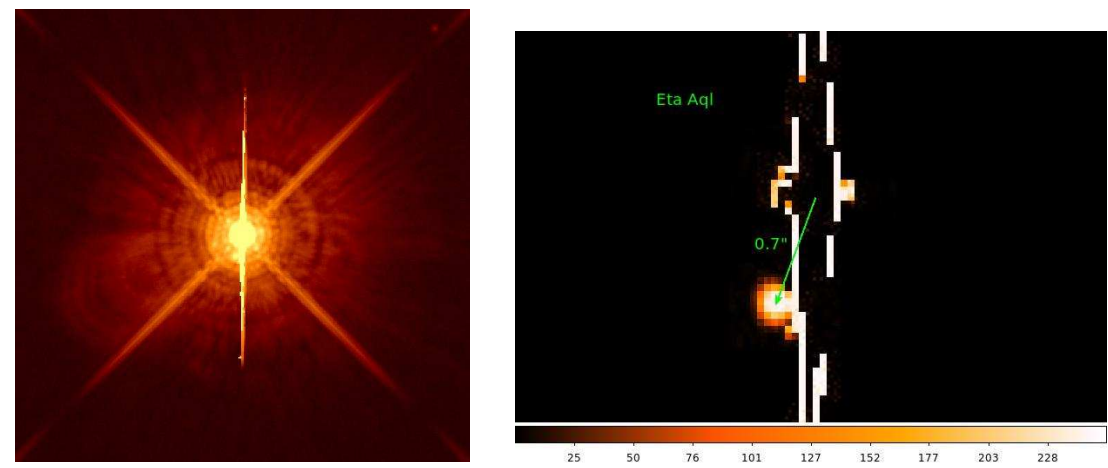

Fig. 1 Left: The center of the HST WFC3 V image of $\eta$ Aql. The image has a log scale and is approximately $10^{\prime \prime}$ wide. Right: The difference image with the Cepheid T Mon subtracted from the $\eta$ Aql image. The companion is clearly visible $0.7^{\prime \prime}$ from the Cepheid. Only the vertical column bleeding remains uncorrected.

\section{Star Formation}

The formation of binary/multiple systems is a very effective way to manipulate angular momentum as a cloud collapses and stars are formed. Thus the distribution of binary parameters provides "fingerprints" of star formation, although, of course, some parameters may subsequently be altered by interactions with other stars. For solar-mass stars, binary and multiple properties have been well characterized, particularly in the seminal study of Duquennoy and Mayor [1] which combined CORAVEL radial velocities with visual binaries and common proper motion pairs. It was recently updated by Raghavan et al. [2] to include recent high resolution techniques. For more massive stars binary properties are less well determined, since they are a rarer group (hence more distant) and have broader lines (hence less accurate radial velocities). Of particular interest among the binary characteristics of intermediate/high mass stars are the frequency of binary and multiple systems, the distribution of mass ratios, and the distribution of separations (including the maximum separation). Cepheids ( $5 \mathrm{M}_{\odot}$ stars) provide a number of tools for investigating binary properties, particularly using multiwavelength techniques. The next sections describe several studies which have the over-arching goal of comparing their binary properties with those of low-mass stars.

HST Survey: We are conducting a survey of 69 of the brightest/nearest Cepheids with the Hubble Space Telescope (HST) Wide Field Camera 3 (WFC3), obtaining images in filters which transform to $\mathrm{V}$ and $\mathrm{I}$. The goal is to look for companions as close as 100-200 AU. The fields typically cover approximately $0.1 \mathrm{pc}=20,000 \mathrm{AU}$, a standard expectation for the maximum dimension of binaries.

HST Survey: $\eta$ Aql has been known since an early IUE (International Ultraviolet Explorer Satellite) observation to have a hot companion. However no evidence of orbital motion has been found in extensive radial velocity observations. Fig. 1 (left) 
shows the image of the Cepheid $\eta$ Aql, displaying a complex point spread function (PSF). Comparison with other WFC3 images revealed the companion, resolved at about 7 o'clock. Work is in progress on PSF correction. However for this relatively bright companion, we started with a simple subtraction. When the image of the Cepheid T Mon (scaled and aligned) was subtracted (Fig. 1 right), the companion was revealed clearly.

HST Survey: Low-Mass Companions. The most difficult companions to identify are low-mass companions with a small mass ratio relative to the primary. The second goal of the HST survey is to identify possible low-mass resolved companions. Fig. 2 (left) provides as an example, the $\mathrm{V}$ image of $\mathrm{R}$ Mus. Possible companions in the field have been identified. The V-(V-I) color magnitude diagram (Fig. 2 right) shows that only one star has the appropriate color-magnitude combination to be a likely companion.
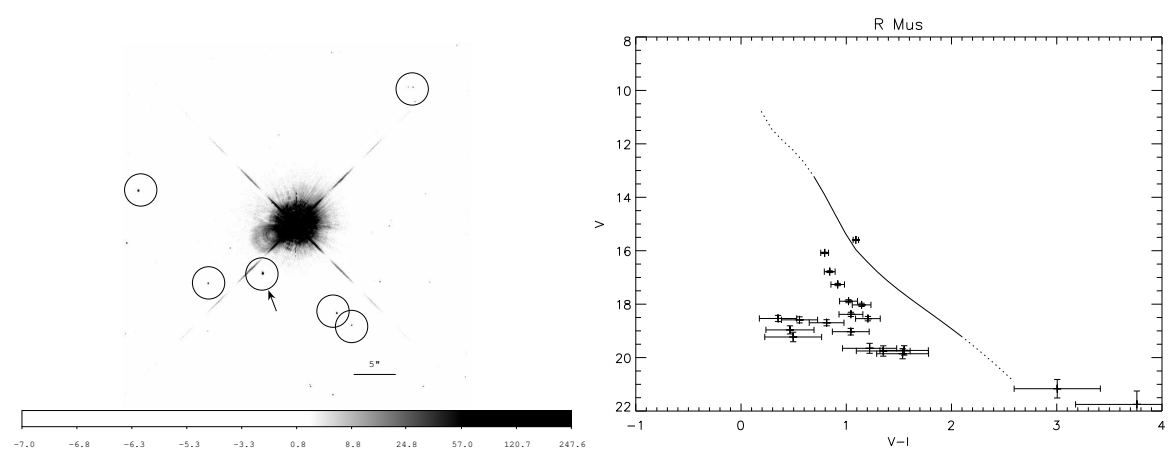

Fig. 2 Left: The V image of R Mus. The brighter stars among the possible companions have been circled, and the arrow indicates the probable companion from Fig. 2 (right). Right: The V(V-I) diagram for faint stars in the R Mus field. The solid line is a ZAMS with the distance and reddening appropriate to the Cepheid. Dotted segments indicate stars too hot (hotter than mid-F spectral type) to produce X-rays or so cool that X-rays would be very difficult to detect.

X-Rays from Low-Mass Companions. Another approach to determining the frequency of low-mass companions of high/intermediate mass stars uses X-ray observations. Late B stars (as well as A stars) do not in general emit X-rays themselves, hence the X-rays that are occasionally found at the location of late B stars are thought to be produced by low-mass X-ray active companions (stars later than mid-F spectral type). We have developed a list of late B stars in the Carina Nebula cluster $\operatorname{Tr} 16$ based on photometry and proper motions [4]. The positions of these stars were compared with the locations of X-ray source in a Chandra ACIS image of the cluster, and the results are shown in Fig. 3. From the fraction of X-ray detected stars, we find that 39 percent of late B stars have low-mass companions with masses between 1.4 and $0.5 \mathrm{M}_{\odot}$. We note that a roughly equal fraction of high mass companions were detected in an IUE survey of Cepheids [3], making the combined fraction of $5 \mathrm{M}_{\odot}$ stars with companions approximately three quarters. 


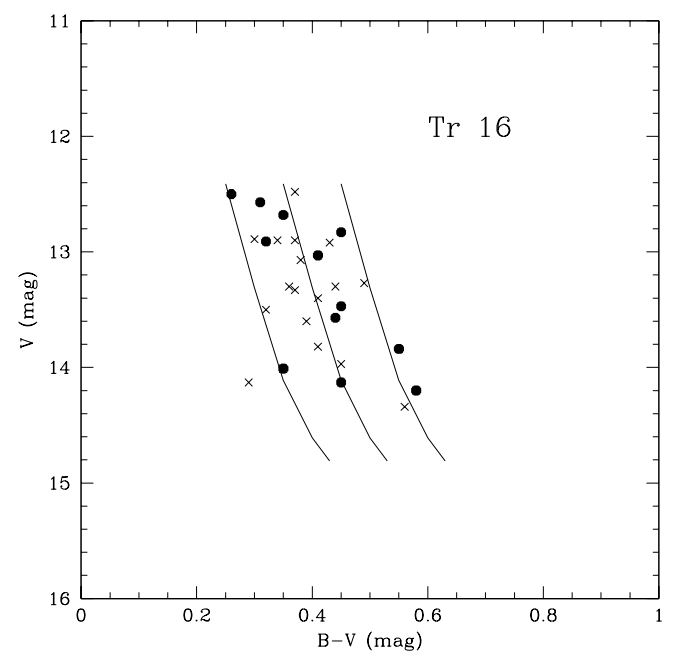

Fig. 3 The V-(B-V) colour-magnitude diagram of the late B stars in Tr 16 based on Cudworth proper motions. Solid lines are the ZAMS with the distance and reddening of $\operatorname{Tr} 16$ (center) with a range in $\mathrm{E}(\mathrm{B}-\mathrm{V})$ of $\pm 0.1 \mathrm{mag}$ (left and right), which is the estimated dispersion in reddening in the cluster. Dots are X-ray sources; crosses are stars not detected in X-rays. Reprinted from ApJS, 194, 13.

\section{Stellar Evolution: Masses}

Observed mass determinations are based on spectroscopic orbits. Considerable new velocity data for Cepheids are available from sources such as the Automated Spectrographic Telescope (AST; Joel Eaton) and the Moscow University group. As an example, see the recent orbit of V350 Sgr [5]. We also have a related ongoing project to use such data sources to search for low amplitude, long period orbits of Cepheids.

Acknowledgements Financial assistance for this work was provided by Hubble grant GO-12215.01A and the Chandra X-ray Center NASA contract NAS8-03060. Fig. 1 was prepared with the help of Howard Bond and Fig. 2 with the help of Evan Tingle.

\section{References}

1. Duquennoy A., Mayor M., 1991, A\&A, 248, 485

2. Raghavan D., et al., 2010, ApJS, 190, 1

3. Evans N. R., 1992, ApJ, 384, 220

4. Evans N. R., et al., 2011a, ApJS, 196, 13

5. Evans N. R., et al., 2011b, AJ, 142, 87 